\title{
Computational aerodynamics of baseball, soccer ball and volleyball
}

\author{
Pouya Jalilian, Patrick K. Kreun, MohammadHady M. Makhmalbaf, William W. Liou
}

Department of Mechanical and Aerospace Engineering, Western Michigan University, Kalamazoo, MI 49008, USA

\section{Email address:}

pouya.jalilian@wmich.edu (P. Jalilian), patrick.k.kreun@wmich.edu (P. Kreun),

m.molabaghermakhmalbaf@wmich.edu (M. Makhmalbaf),william.liou@wmich.edu (W. Liou)

\section{To cite this article:}

Pouya Jalilian, Patrick K. Kreun, MohammadHady M. Makhmalbaf, William W. Liou. Computational Aerodynamics of Baseball, Soccer Ball and Volleyball. American Journal of Sports Science. Vol. 2, No. 5, 2014, pp. 115-121. doi: 10.11648/j.ajss.20140205.12

\begin{abstract}
Recent advances in the computing power of modern computers have made computational fluid dynamics studies particularly interesting and feasible. We used the computational fluid dynamics method to solve the physical governing equations of the air flow around balls of popular sports in typical game conditions and investigated their aerodynamics and the flight characteristics. The work presented here describes the construction of the computational fluid dynamics models for a baseball, volleyball and two soccer balls, and the use of these models to analyze the effects of spin rate, surface pattern, and size for their respective sports. The computational results show significant correlations between ball spin rate and the aerodynamics forces, including drag and lift, for soccer ball, baseball and volleyball. For the baseball, the lift and lateral forces are shown to have also been influenced by the lace orientation.
\end{abstract}

Keywords: CFD, Baseball, Soccer Ball, Volleyball

\section{Introduction}

Aerodynamics in sports has attracted attention of scientists and researchers for centuries. The analysis of sporting ball aerodynamics can be traced to Newton, 1672, who studied and reviewed the flight of a tennis ball. A number of experimental and model-based techniques have been employed in studying the flight of sporting balls.

Recent advances in the field of computational fluid dynamics (CFD) have led to a dramatic rise in the pace and relative ease in which these studies can be conducted. The flexibility of CFD lends itself well to be used in investigations of a broad variety of subjects in sports. For example, CFD simulations can help understand the extraordinary motions of soccer ball, or identify the ideal lace orientation to provide the desired trajectory of a baseball pitch. These types of studies are also of interest to sports equipment manufacturers striving to advance the performance characteristics of their products.

This paper will present the computational aerodynamic analysis of two different soccer balls, a baseball and a volleyball. A commercial CFD package has been used. We first compute the aerodynamics of these balls in their typical play conditions to serve as a baseline data for comparisons.
The resulting trajectories have also been evaluated and will be presented. We then perform case studies to examine the aerodynamic forces exerted on each ball for a variety of linear and angular velocities, and ball sizes, that are prompted by how the balls are, or can be, played.

\subsection{Literature Survey}

Advancements in sporting equipment have been fueled not only by the competitive nature of sport, but also a greater understanding of the physics governing the way sports are played. Metha (1985) devised a series of experiments on cricket balls, baseballs and golf balls. Aerodynamic forces were obtained and reported in terms of the spinning rate of the balls. Pallis and Mehta (2002) performed wind tunnel studies for sporting balls. Asai, Ito, Seo and Hitotsubashi (2010) showed that the critical Reynolds number at which the drag force drops dramatically is remarkably smaller for new types of volleyball, resulting in faster ball speed. In other studies (Mizota et al., 2013; Oggiano \& Saetran, 2010; Barber \& Carré, 2010; Goff \& Carré, 2009, 2010; Asai, Seo, Kobayashi, \& Sakashita, 2007; Carré, Asai \& Haake 2002), wind tunnel tests and trajectory analyses were conducted for different types of soccer balls, including a spinning 32-panel soccer ball that is akin to the generic soccer ball used in this 
study. Goodwills, Chin and Haake (2004) performed a similar study and compared spinning and non-spinning tennis balls. Barber, Chin, and Carré (2009) used CFD simulation to study aerodynamic behavior of soccer balls. Alam, Chowdhury, Stemmer, Wang, and Yang (2012), and Alam et al. (2011) conducted experiments in order to study the aerodynamic forces of several commercially available soccer balls. Similar studies were performed by Alam et al. (2011) and Alam et al. (2012) on baseballs.

\section{Methods}

The flow of air around the sporting balls presented in this study is governed by the Navier-Stokes equations, the solutions of which describe the flow field around the sports balls, and thus their aerodynamics characteristics, such as lift and drag. A numerical method for solving the Navier-Stokes equations involves the use of computer to integrate the differential equations and yield a solution. This approach is commonly referred to as CFD and has grown in popularity with the continuing advancements of computing power. The ANSYS FLUENT 14 software has been applied in various industries and is used in this study. The creation of a CFD model for each ball requires first building a three-dimensional digital model for both the ball and surrounding field of air. These models are then meshed into discrete sub-volumes defining the numerical domain and the appropriate initial and boundary conditions needed for each case being evaluated. These steps are described in the following sections.

\subsection{Geometry}

Geometric features play an important role in determining the aerodynamic characteristics of the sporting balls. Manufacturers of soccer balls and volleyballs can vary, to an extent, patch patterns, seam designs and surface roughness. They influence the ball's behavior during flight. For the balls used in this study, the more crucial surface features to investigate are seams, laces and patch patterns. To this end, each ball has been carefully modeled to accurately capture these fine, but aerodynamically important surface features.

\subsubsection{Soccer Ball and Volleyball}

The regulation volleyball with a diameter of $210 \mathrm{~mm}$ and two different soccer balls, namely, a generic soccer ball with $220 \mathrm{~mm}$ diameter and the 86-panel soccer ball with $225 \mathrm{~mm}$ diameter have been selected for study. In the history of soccer, balls of different pattern, size and weight have been used. We have selected two different designs to study the effects of the geometric features of soccer balls. Their geometries were obtained from an open source solid modeling website, GrabCAD (Thompson, 2012; Niven, 2012). Minor irregularities in the geometry files, such as holes and gaps, are removed. Figure 1( $a, b$ and $c)$ illustrates the final geometries used in the CFD simulations.

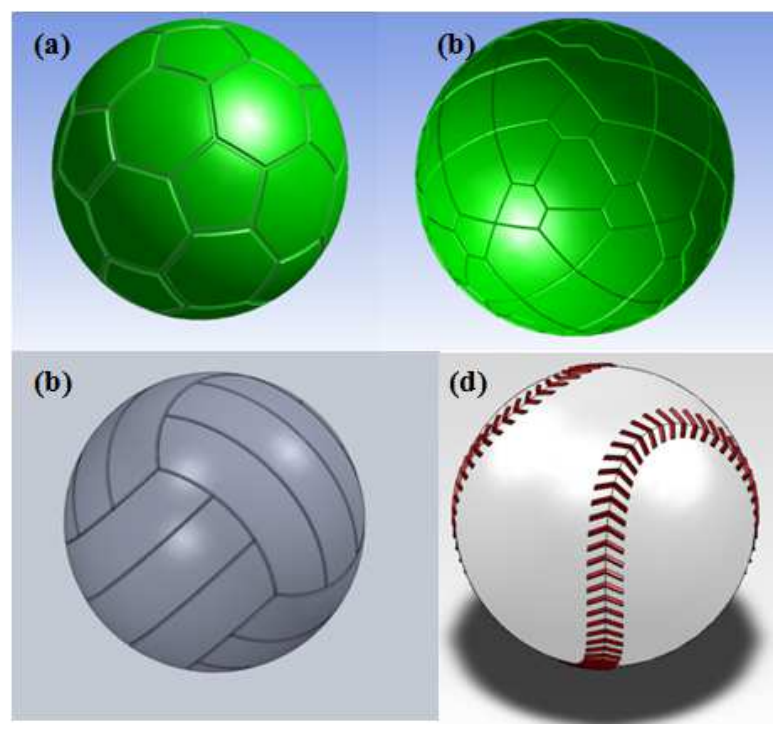

Figure 1. The generic soccer ball geometry with $220 \mathrm{~mm}$ diameter (a), the 86-panel soccer ball geometry with $225 \mathrm{~mm}$ diameter (b), the volleyball geometry with $210 \mathrm{~mm}$ diameter (c) and the baseball geometry with 73 mm diameter $(d)$.

\subsubsection{Baseball}

We created the geometry files for the baseball model using a computer-aided-engineering software. A sphere, 73 $\mathrm{mm}$ in diameter was first generated. Then, a three-dimensional sketch was added to the sphere defining the seam pattern. Finally, by generating additional sketch planes, one lace was added to the surface of the sphere and copied using a curve driven pattern command to produce the 108 individual stitches on the surface of the ball, as is shown in Figure 1-d.

\subsection{Meshing Generation}

Once the geometry was created, the next step was mesh generation in a computational domain, where a computer solution of the Navier-Stokes equations will be sought. Basically, the computational domain must be split into small elements or cells, the collection of which comprises a mesh. Then, the governing equations are discretized for these elements where solutions will be obtained.

\subsubsection{General Mesh Structure}

Following the successful mesh structure employed by Barber et al. (2009), the general mesh structure we set up is shown in Figure 2.

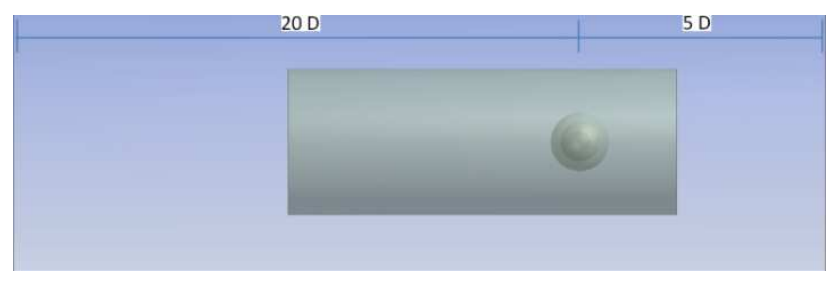

Figure 2. The chosen general mesh zones including a small sphere, a larger sphere, a cylinder and the enclosure. The ball center was placed 5 diameters from the inlet, 20 diameters from the outlet and 5 diameters from the outer domain boundary sides. 
A ball geometry was imported and a computational domain for the fluid was constructed around the ball by applying a Boolean operation to subtract the ball volume from the surroundings (air). This results in the computational domain for the fluid with a matched fluid-ball boundary. To allow a greater control over the sizes of the mesh elements, three intermediate regions have been used in the computational domain, as illustrated in Figure 2. They include (1) a small sphere immediately surrounding the sporting ball, (2) a larger sphere encompassing the sporting ball and the first sphere, and (3) a cylinder which encloses the ball and the two spheres. The size of the computational domain has been determined in such a way that it reduces the effects of the placement of the outer computational boundaries on the computed flow near the ball.
The process of generating the mesh structures has been utilized for the soccer ball and volleyball, and is only a slightly different from that for the baseball.In this study, the tetrahedron meshing method was used. For the sphere-shaped intermediate regions, the mesh sizes are configured to provide the greatest resolution of the fluid-ball boundary. This is necessary to properly capture the viscous effects of the flow around the rough patches and seams. Meshes were also refined in the cylindrical intermediate region to resolve the flow in the wake of the ball. In the case of the baseball, an additional surface refinement has been applied to the laces to provide sufficient resolution in these regions to capture small-scale flow structures. Figure 3 highlights the finer resolution around the seams and laces for, respectively, soccer ball, volleyball, and baseball.
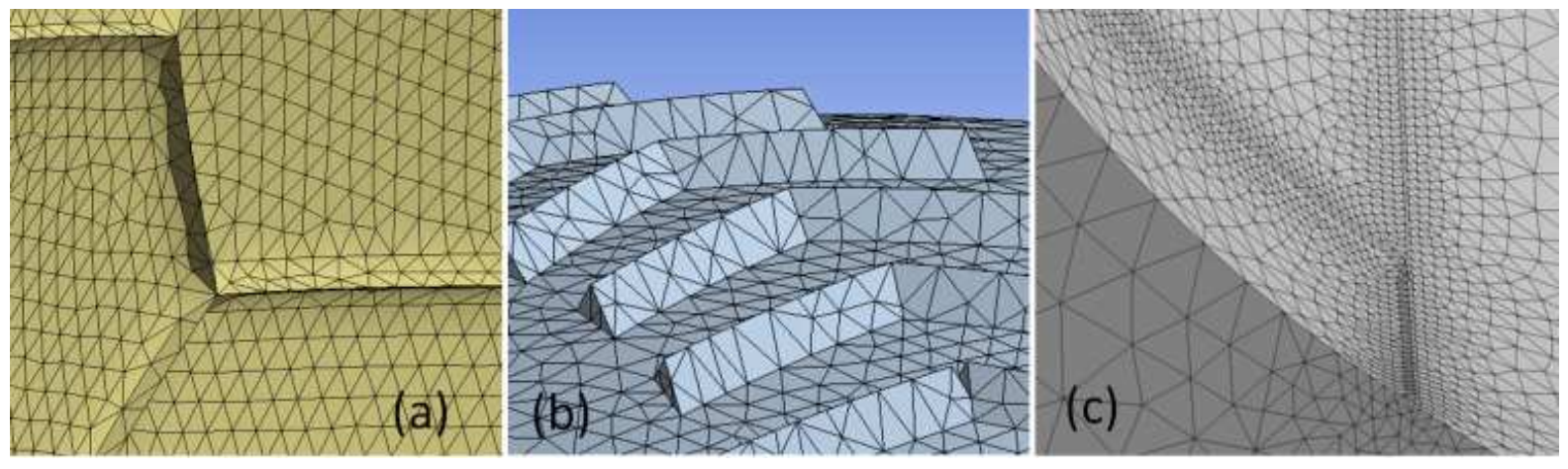

Figure 3. Meshing near the seams of (a) the 86-panel soccer ball, (b) baseball and (c) volleyball.

\subsubsection{Mesh Quality}

To enhance accuracy of the numerical solution, the mesh quality was evaluated. The mesh quality metrics used include the element quality, aspect ratio, Jacobian ratio, warping factor, parallel deviation, maximum corner angle, skewness and orthogonal quality. Each of these quality metrics was assessed for the different sporting ball models and confirmed in accordance with the software's guidelines.

\subsection{Setup}

After establishing quality meshes, the various CFD runs were properly setup. For this study, a pressure-based, steady-state solver was employed. To include the effect of turbulence in the flow, a realizable k- $\varepsilon$ turbulence model was chosen with standard wall treatments. The standard wall-function, near-wall treatment was selected in order to reduce the number of mesh element required near solid surfaces and, thus, the overall mesh counts.

\subsubsection{Boundary Conditions}

A crucial aspect of properly setting up a CFD model is the definition of the boundary conditions. The primary boundary conditions to consider here are the fluid inlet, fluid outlet, outer fluid boundary, and ball-fluid interface. It should be noted that, although each ball travels with a velocity through the fluid in the physical system, this was modeled as a stationary, rotating ball with the fluid passing around it. In keeping with this change of reference of frame, the inlet (see Figure 2 for reference) was defined having a velocity, normal to the boundary, matching with that specified for the corresponding case being examined. Turbulence at this boundary was specified as having between $0.5 \%$ and $1 \%$ intensity. A $0.5 \mathrm{~m}$ length scale was used, which described low levels of turbulence in the air with relatively large eddy structures, comparing with the sizes of the balls studied. The fluid outlet was described similarly with like turbulence values; however, rather than a velocity, a gauge pressure of 0 Pascal was assigned.

To describe the rotational motion, a moving mesh boundary condition was applied. The moving mesh boundary condition allows the two meshed bodies to slide relative to each other, thereby capturing properly the effects of the rotating surface features, such as laces, seams and patches. The resulting non-conformal mesh is handled by passing fluxes using the non-conformal interface algorithm. An angular velocity about the axis of rotation was specified to match the angular velocity of the corresponding case. The surface roughness of each ball was used to match that of its physical counterpart.

A no-slip shear was applied at the ball surface (wall) and a free-slip shear condition was applied on the outer boundaries of the computational domain.

\subsubsection{Solution}

The solution process was initialized using the inlet boundary conditions as the initial velocity throughout the fluid domain. Second-order upwinding schemes were 
employed for the momentum, turbulent kinetic energy, turbulent dissipation, and energy equations.

\subsection{Trajectory Analysis}

In previous studies (Goff \& Carré, 2009, 2010; Carré et al., 2002), sporting ball trajectory was determined from experimetal data. The measured trajectory was then used to obtain drag and lift coefficients. In contrast, in this study, the trajectory is predicted based on the CFD simulated drag and lift forces, and therefore, reflects the simulated aerodynamics of the ball. Figure 4 illustrates a free-body diagram of a ball subjected to the drag, lift and gravitational forces. The ball spins around the horizontal axis perpendicular to the ball's velocity vector and the side force is neglected. With the Newton's second law, the equation governing the motion of the ball can be written as

$$
\boldsymbol{F}_{\boldsymbol{D}}+\boldsymbol{F}_{\boldsymbol{L}}+m \boldsymbol{g}=m \boldsymbol{a}
$$

where $m$ is the mass of the ball, $\boldsymbol{g}$ the vector gravitational acceleration, $\boldsymbol{a}$ ball's vector acceleration. $\boldsymbol{F}_{\boldsymbol{D}}$ and $\boldsymbol{F}_{\boldsymbol{L}}$ are drag and lift force vectors, respectively, the magnitudes of which are defined as $F_{D}=0.5 C_{D} \rho A v^{2}$ and $F_{L}=0.5 C_{L} \rho A v^{2}$, where $\rho$ denotes the air density, $A$ the ball's cross sectional area and $v$ the ball's velocity magnitude. The $\mathrm{x}$ and y components of (1) may be written as

$$
\begin{gathered}
-\rho A v\left(C_{L} v_{y}+C_{D} v_{x}\right)=2 m x^{\prime \prime} \\
\rho A v\left(C_{L} v_{x}-C_{D} v_{y}\right)-2 m g=2 m y^{\prime \prime}
\end{gathered}
$$

where $v_{x}=v \cos \theta, v_{y}=v \cos \theta, x^{\prime \prime}=d^{2} x / d t^{2}$ and $y^{\prime \prime}=d^{2} y / d t^{2}$. $\theta$ denotes the angle between the ball's velocity vector and the horizontal line (x axis), as shown in Figure 4.

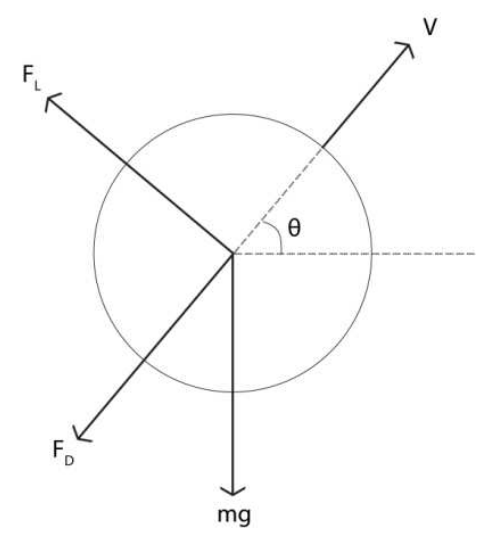

Figure 4. The free-body diagram of a sporting ball spinning around the axis perpendicular to the page (side view)

The above system of equations consists of two second order nonlinear ordinary differential equations. The fourth-order Runge-kutta method has been used in a Mathcad (15th version) code to solve the above equations numerically for the $\mathrm{x}$ and $\mathrm{y}$ coordinates of the ball and, thus, the trajectory. The drag and lift coefficients are assumed constant throughout the ball flight.

\section{Results}

To study the effects of the prominent features of the balls on their aerodynamics and flights, a number of test cases were developed. Computer simulations of these cases were subsequently performed and the results were analyzed.

\begin{tabular}{|c|c|c|c|c|c|c|c|c|c|}
\hline \multirow[b]{2}{*}{ Case } & \multicolumn{3}{|c|}{ Volleyball } & \multicolumn{3}{|c|}{ Soccer ball (The generic and 86 -panel) } & \multicolumn{3}{|l|}{ Baseball } \\
\hline & Size & $\operatorname{Re}\left(\times 10^{3}\right)$ & Spin Rate & Size & $\operatorname{Re}\left(\times 10^{3}\right)$ & Spin Rate & Size & $\operatorname{Re}\left(\times 10^{3}\right)$ & Spin Rate \\
\hline 1 & Actual & 250 & $5 \mathrm{~Hz}$ & Actual & 250 & $5 \mathrm{~Hz}$ & Actual & 250 & $50 \mathrm{~Hz}$ \\
\hline 2 & Football & 330 & $5 \mathrm{~Hz}$ & Actual & 330 & $5 \mathrm{~Hz}$ & Football & 330 & $5 \mathrm{~Hz}$ \\
\hline 3 & Actual & 250 & $10 \mathrm{~Hz}$ & Actual & 250 & $10 \mathrm{~Hz}$ & Actual & 250 & $25 \mathrm{~Hz}$ \\
\hline 4 & ------- & ------- & ------- & Actual & 250 & $25 \mathrm{~Hz}$ & Actual & 250 & $25 \mathrm{~Hz}^{*}$ \\
\hline
\end{tabular}

\subsection{Test Cases}

Table 1. Description of run conditions

*The rotation axis is a horizontal line perpendicular to the line of the air flow for each case. However, the spin axis was rotated 30 degrees with respect to the vertical line (perpendicular to the air flow line) in case 4 for the baseball in order to show the effect of the lace orientation.

Table 1 presents the cases simulated with varying ball sizes, Reynolds numbers, and spin rates. Each row reflects a set of conditions for the corresponding ball models that highlights a particular feature of the ball, or an in-game scenario. In Case 1, the values of the variables represent that of a typical in-game condition for each sport. Case 2 is designed to compare the effects of the surface pattern and shape on the resulting aerodynamic forces by using an identical diameter (as that of the soccer ball) for all the balls. In Case 3, we vary the typical spin rates seen in the respective games, as depicted in Case 1. Specifically, the spin rates for soccer ball and volleyball were doubled, and that of the baseball halved. Case 4 describes a made-up condition in which the spin rate normally seen in a baseball pitch is applied to a soccer ball. This particular numerical experiment is designed to demonstrate the effects of the size and the surface pattern for a given spin rate.

\subsection{Test Results}

Table 2. Drag and lift coefficients computed from test cases

\begin{tabular}{lllllll}
\hline Case & \multicolumn{2}{l}{ Volleyball } & \multicolumn{2}{l}{$\begin{array}{l}\text { Soccer ball (The } \\
\text { generic) }\end{array}$} & \multicolumn{2}{l}{ Baseball } \\
\hline & $\boldsymbol{C}_{\boldsymbol{D}}$ & $\boldsymbol{C}_{\boldsymbol{L}}$ & $\boldsymbol{C}_{\boldsymbol{D}}$ & $\boldsymbol{C}_{\boldsymbol{L}}$ & $\boldsymbol{C}_{\boldsymbol{D}}$ & $\boldsymbol{C}_{\boldsymbol{L}}$ \\
\hline 1 & 0.185 & 0.213 & 0.211 & 0.116 & 0.417 & 0.141 \\
2 & 0.096 & 0.104 & 0.199 & 0.095 & 0.081 & 0.025 \\
3 & 0.223 & 0.216 & 0.235 & 0.181 & 0.410 & 0.102 \\
4 & ----- & ------ & 0.331 & 0.289 & 0.410 & 0.085 \\
\hline
\end{tabular}


Table 2 presents the simulated drag coefficient $C_{D}$ and lift coefficient $C_{L}$ for all the cases. Comparing the results of Cases 1 and 3, it can be seen that for the generic soccer ball and volleyball, the higher spin rate in Case 3 increases $C_{D}$ and $C_{L}$. For the baseball, the lower spin rate in Case 3 decreases both $C_{D}$ and $C_{L}$ from those of Case 1 .

Figure 5 displays the air pressure contours in the vicinity of each ball studied for Case 3. There are noticeable effects of the rotation and ball size on the pressure fields. It is clear that for a ball with a smaller size and higher spin rate, larger pressure differential can been generated, resulting in a larger net force perpendicular to both the axis of rotation and the air flow. Figure 6 shows the streamlines of the simulated air flow around each ball for Case 3. The ball's wake is more clearly shifted with higher spinning rate.

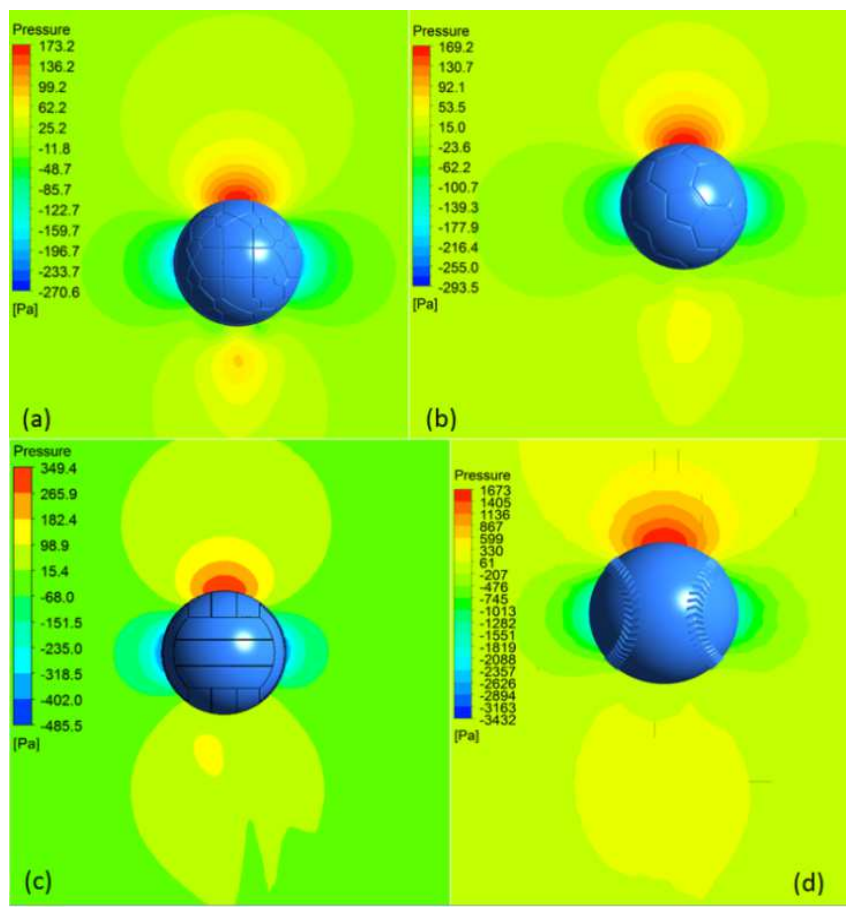

Figure 5. Pressure contours corresponding to Case 3 for the (a) 86-panel, (b) and generic soccer ball, (c) volleyball and (d) baseball.

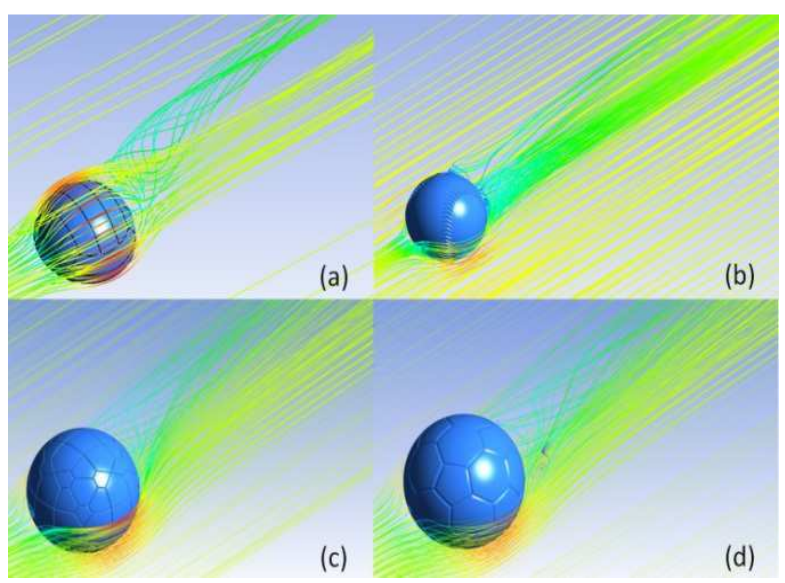

Figure 6. The streamlines corresponding to Case 3 for (a) volleyball, (b) baseball, (c) the 86-panel football and (d) the generic football.
The surface shear stress distributions on the balls' surface reveal the importance of seams, patches and surface features. Figure 7 demonstrates that for Case 3 , in regions near the seams, wall shear stress changes locally due to the localized small flow structures. This feature of the surface shear stress distribution affects the aerodynamic drag and lift, indicating the potential importance of the ball patterns in the playing and the further development of these sports. Also clear from the figure, the stagnation point, the point on the leading surface of the ball at which the shear stress is zero, is shifted left. This anticipated shift, due to the rotation of the ball is more apparent in the case of baseball where the applied spin rate is the highest.

Using the data collected by Asai et al. (2010), we compared the predicted drag coefficients for the volleyball with the test results. The results showed agreement to within $15 \%$. We also compared the predicted drag coefficients of the modelled generic 32-panel soccer ball with those in previous studies (Barber \& Carré, 2010; Goff \& Carré, 2009; Asai et al., 2007). The computer simulations under-predict the drag coefficients by about $10 \%-15 \%$. One of the reasons for the computed drag to digress from test results is likely due to the fact that the simulations are predicted assuming a fully turbulent flow around the ball and use a turbulence model accordingly. In field tests, it has been shown that the air flow around the ball can often be a combination of turbulent and laminar flow in the supercritical region and is not fully turbulent (Barber et al., 2009).

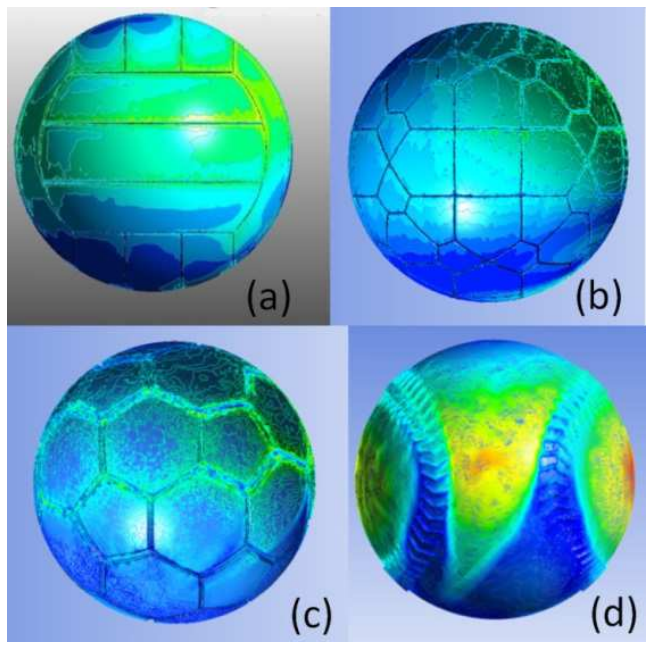

Figure 7. The surface shear stress distributions corresponding to Case 3 for (a) volleyball, (b) the 86-panel soccer tball, (c) the generic soccer ball and (d) baseball.

We calculated the trajectory of the generic soccer ball with $\rho=1.225 \mathrm{~kg} . \mathrm{m}^{-3}, g=9.81 \mathrm{~m} . \mathrm{s}^{-1}$. For the generic soccer ball, $m=0.430 \mathrm{~kg}$ and $A=0.0380 \mathrm{~m}^{2}$. Also, $\theta=20^{\circ}$ at $t=0 \mathrm{~s}$ is assumed. The trajectory results are shown in Figure 8. The results indicate that a higher ball's velocity results in a longer horizontal distance a ball can travel and a higher spin rate yields a taller trajectory. 


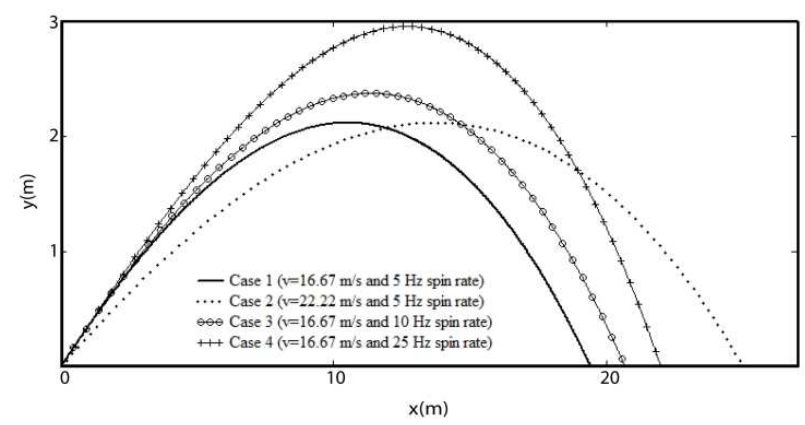

Figure 8. Computed trajectories for the generic soccer ball.

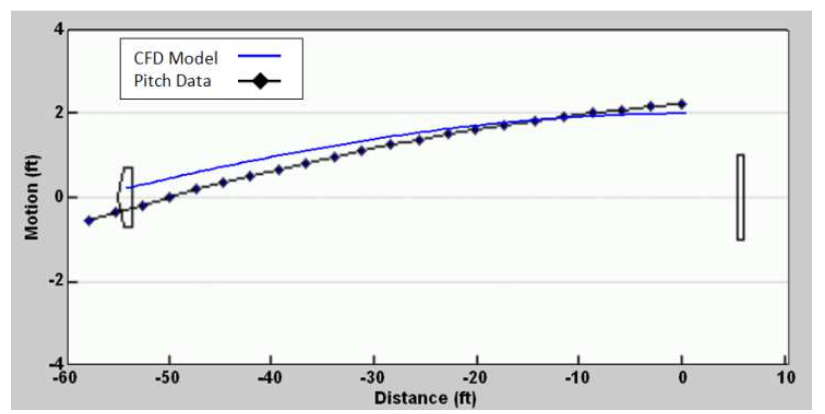

Figure 9. Comparison of the CFD computed baseball trajectory with the actual pitch data from Somers (2013).

For the baseball, there exists a vast collection of in-game pitch data recorded by modern pitch tracking systems. A proper reduction of these data, when averaged over a wide range of conditions, for example, provides important information that can be used for validation of computer simulations. For the validation of the baseball simulations, the pitch motion resulting from the model predicted drag and lift forces was compared with the actual pitch motion of an American pitcher, Justin Verlander, of the Detroit Tigers. Curveball data from May 1, 2011 to October 25, 2011 by compiled Somers (2013) were averaged to yield an average spin rate, linear velocity, and trajectory. Using the measured spin rate and the linear velocity as inputs to the CFD model, we compare the total pitch motion and the trajectory results of the simulation with the measurement. The result for the trajectory comparison is shown in Figure 9. As evidenced by Figure 9, the predicted trajectory closely matches with the actual pitch data.

\section{Discussion}

The results of simulations shown above indicate that both the drag and lift coefficients are highly dependent on the rotational and the linear motions of the ball. For discussions, we employ a non-dimensional spin parameter, $S_{p}=r \omega / v$ where $r$ denotes the ball radius (m), $\omega$ the angular velocity $\left(\right.$ rad. $\left.^{-1}\right)$ and $v$ the linear velocity $\left(m . s^{-1}\right)$. The simulated results obtained here indicate that increasing the value of $S_{p}$ results in an increase of both the drag and the lift coefficients. In other words, the faster the ball rotates, the more drag and lift forces are exerted on the ball. Not too surprisingly, with decreases of the spin parameter, the predicted lift and drag forces decrease for a given linear velocity. Therefore, it could be advantageous for players to kick the ball to a small spin rate in games, which will result in a lower drag force and, therefore, a longer distance. Naturally, the stabilizing effects of the spinning can decrease with a reduced spin rate, which, in turns, compromises the accuracy of a long kick. Additionally, the results indicate that, compared to the generic soccer ball, the 86-panel soccer ball has higher drag and lift coefficients due to the increased number of panels. There are apparent effects of the geometric features on the aerodynamic characteristics of soccer balls.

In the case of the baseball, the spin parameter has been shown to have a less significant impact on the drag, but a very strong effect on the lift and the overall motion of the pitch. This would imply there is not a significant trade-off, from an aerodynamics standpoint, between linear pitch speed and angular velocity, as it is for soccer ball. In fact, the most advantageous situation would appear to be the case of greatest spin rate. However, it is also necessary to account for the varying human body mechanics between different pitches, which generally dictate the maximum pitch speed achievable. We also highlighted the importance of lace orientation with respect to the total aerodynamic forces generated. Not only does the lace orientation assist a pitcher in applying spin to a pitch, but the laces themselves have a substantial impact on the total motion of a pitch. This study showed that, for a 30 degree change in the lace orientation, the aerodynamic force responsible for the lateral motion dropped by approximately $17 \%$ with a $7 \%$ increase in the lift.

The travel distance of a volleyball during an actual game can be no longer than 20 meters, which is the diagonal length of a regulation volleyball court. The typical time in the air for a fast ball is about 0.7 seconds. A player must move into position behind the ball during this short period of time to make a play. Wobbling and unpredictable behavior of the ball caused by its axisymmetric surface patterns add to the difficulty for a player to anticipate the ball's trajectory and react accordingly. The critical ball speed associated with the critical Reynolds number, at which the drag coefficient drops dramatically, is an important aerodynamic parameter. For a ball travelling near the critical Reynolds number, the magnitude of the drag force is relatively small and has the least effect on the ball. This makes the motion of the ball smoother and more predictable. The lower drag also increases the ball's speed and consequently the pace of the game. Therefore, it can be advantageous to design the volleyball so that the critical ball speed is close to the speed range of real-world plays. It is shown in Table 2 that the volleyball in Case 2 has small drag and lift coefficients compared to those in Cases 1 and 3. It suggests that the critical Reynolds number is between 250,000 and 330,000, which is in agreement with the results of Asai et al. (2010).

\section{Conclusions}

This work presented the processes and the results of a computational study of the aerodynamics of soccer ball, 
baseball and volleyball. We simulated their aerodynamic behaviors and identified a number of important aerodynamics features of each ball. The analysis showed that for the soccer ball and volleyball, the spin parameter plays an important role in determining the resulting aerodynamic forces, provided the Reynolds number is above 200,000. At this point, the lift and drag forces become closely tied to the spin parameter and largely independent of the Reynolds number. Conversely, it was found that, for the baseball, the drag coefficient was not as closely related to the spin parameter, but the forces that generate the vertical and lateral motions were. The orientation of the laces was also shown to have a strong influence on the total aerodynamic forces generated. Finally, by comparing soccer balls of differing patch designs, the ball with the greater number of patches, and thus the greater number of seams, was found to yield higher drag and lift coefficients. Computational aerodynamics methods reported here can be readily applied to other sports. We would recommend future efforts to couple computational and experimental studies to better align the conditions used in the two approaches and to better integrate their merits toward actual playing of the sports.

\section{References}

[1] Alam, F., Chowdhury, H., Moria, H., Fuss, F. K., Khan, I., Aldawi, F., \& Subic, A. (2011). Aerodynamics of contemporary FIFA soccer balls. Procedia Engineering, 13, 188-193.

[2] Alam, F., Ho, H., Chowdhury, H., \& Subic, A. (2011). Aerodynamics of baseball. Procedia Engineering, 13, 207-212.

[3] Alam, F., Chowdhury, H., Stemmer, M., Wang, Z., \& Yang, J. (2012). Effects of surface structure on soccer ball aerodynamics. Procedia Engineering, 34, 146-151.

[4] Alam, F., Ho, H., Smith, L., Subic, A., Chowdhury, H., \& Kumar, A. (2012). A study of baseball and softball aerodynamics. Procedia Engineering, 34, 86-91.

[5] ANSYS, Inc. (2010) ANSYS Meshing User's Guide

[6] Asai, T., Seo, K., Kobayashi, O., \& Sakashita, R. (2007). Fundamental aerodynamics of the soccer ball. Sports Engineering, 10(2), 101-109.
[7] Asai, T., Ito, S., Seo, K., \& Hitotsubashi, A. (2010). Aerodynamics of a new volleyball. Procedia Engineering, 2(2), 2493-2498.

[8] Barber, S., Chin, S. B., \& Carré, M. J. (2009). Sports ball aerodynamics: a numerical study of the erratic motion of soccer balls. Computers \& Fluids,38(6), 1091-1100.

[9] Barber, S., \& Carré, M. J. (2010). The effect of surface geometry on soccer ball trajectories. Sports Engineering, 13(1), 47-55.

[10] Carré, M. J., Asai, T., Akatsuka, T., \& Haake, S. J. (2002). The curve kick of a football II: flight through the air. Sports Engineering, 5(4), 193-200.

[11] Goff, J. E., \& Carré, M. J. (2009). Trajectory analysis of a soccer ball. American Journal of Physics, 77(11), 1020-1027.

[12] Goff, J. E., \& Carré, M. J. (2010). Soccer ball lift coefficients via trajectory analysis. European Journal of Physics, 31(4), 775 .

[13] Goodwill, S. R., Chin, S. B., \& Haake, S. J. (2004). Aerodynamics of spinning and non-spinning tennis balls. Journal of wind engineering and industrial aerodynamics, 92(11), 935-958.

[14] Mehta, R. D. (1985). Aerodynamics of sports balls. Annual Review of Fluid Mechanics, 17(1), 151-189.

[15] Mizota, T., Kurogi, K., Ohya, Y., Okajima, A., Naruo, T., \& Kawamura, Y. (2013). The strange flight behaviour of slowly spinning soccer balls. Scientific reports, 3 .

[16] Newton, I. (1672). A new theory about light and colors. Philosophical Transactions of the Royal Society, 80, 3075-3087

[17] Niven, L. (2012) https://grabcad.com/library/soccer-ball--2 /files/practice.SLDPRT

[18] Oggiano, L., \& Sætran, L. (2010). Aerodynamics of modern soccer balls.Procedia Engineering, 2(2), 2473-2479.

[19] Pallis, J. M., \& Mehta, R. D. (2002). Aerodynamics and hydrodynamics in sports. The Engineering of Sport, 4, 31-39.

[20] Somers, Trip. (2013). Justin Verlander, Detroit Tigers PITCHf $/ x \quad$ Pitcher Profile. http://pitchfx.texasleaguers.com/pitcher/434378/

[21] Thompson,S.(2012),https://grabcad.com/library/86-panel -soccer-ball design /files / 86\%20panel\%20dome.SLDPRT 